\title{
UMA INVESTIGAÇÃO TEÓRICA SOBRE RELAÇÕES SEMÂNTICAS PARTITIVAS E SUA APLICAÇÃO EM SISTEMAS DE ORGANIZAÇÃO DO CONHECIMENTO
}

\author{
A THEORETICAL INVESTIGATION ABOUT PART- \\ WHOLE SEMANTIC RELATIONS AND ITS \\ APPLICATION IN KNOWLEDGE ORGANIZATION \\ SYSTEMS
}

\author{
Maurício Barcellos Almeida \\ Jeanne Louize Emygdiob
}

\begin{abstract}
RESUMO
Introdução: Relações semânticas são essenciais para a Representação do Conhecimento, um campo de pesquisa que ganha cada vez mais relevância, principalmente a partir do advento da Web Semântica. A Ciência da Informação tem um longo histórico de estudo e uso de relações semânticas nos diversos contextos em que Sistemas de Organização do Conhecimento (SOCs) são concebidos e construídos. Entretanto, são poucos os estudos pormenorizados sobre a relação semântica partetodo, também conhecida como relação partitiva. Objetivo: o presente artigo objetiva uma descrição fundamentada da relação partitiva, a partir de abordagem interdisciplinar que envolve suas origens linguísticas e filosóficas. Metodologia: para atingir o objetivo proposto, descrevem-se e caracterizam-se as duas principais formas pelas quais as relações partitivas se manifestam - a meronímia, não formal e linguística; e a mereologia, formal e lógica - distinguindo-as pela propriedade da transitividade. Resultados: a partir da distinção mencionada, sugere-se critério pragmático ao decidir qual abordagem de relação partitiva é adequada quando se constroem ontologias e tesauros. Conclusões: conclui-se, que as relações formais são, de fato, mais restritas, e pode-se mesmo afirmar que estão incluídas nas relações não-formais, mas que o uso das relações formais em ontologias requer cuidados muitas vezes negligenciadas pelos cientistas da informação.
\end{abstract}

Descritores: Ontologias. Relações semânticas. Relações parte-todo.

\footnotetext{
a Doutor em Ciência da Informação pela Escola de Ciência da Informação da Universidade Federal de Minas Gerais (UFMG). Professor do Programa de Pós-Graduação em Gestão \& Organização do Conhecimento da Escola de Ciência da Informação da Universidade Federal de Minas Gerais (UFMG). E-mail: priv.mba@gmail.com

b Doutoranda em Ciência da Informação pela Escola de Ciência da Informação da Universidade Federal de Minas Gerais (UFMG). E-mail: jeanne.emygdio@gmail.com
} 


\section{INTRODUÇÃO}

Relações semânticas são associações significativas entre entidades, de interesse para a Ciência da Informação, pelo papel essencial que desempenham nos processos que pessoas usam para representar conhecimento. Dentre as relações semânticas, destaca-se uma relação genericamente denominada por parte-todo, que lida, como seu nome sugere, com entidades tratadas como todos e as partes ligadas a elas.

O primeiro passo para entender a relação parte-todo é considerar noções intuitivas que as pessoas naturalmente possuem, as quais desempenham importante papel no sistema conceitual (FLAVELL; MARKMAN, 1983) e na percepção visual humana (BIEDERMAN, 1987). Um simples objeto permite diferentes possibilidades de divisão em partes, de acordo com um esquema perceptivo ou conceitual. Por exemplo, do ponto de vista perceptivo, uma luminária pode ser dividida em base, haste e cúpula; do ponto de vista conceitual, a divisão para a mesma luminária pode considerar partes não aparentes e dessa forma ausentes no esquema perceptivo, por exemplo, lâmpadas, fios, parafusos, interruptores, etc. (PRIBBENOW, 2002).

Ao falar sobre o mundo, as pessoas separam trivialmente as entidades em partes. Entretanto, o significado do termo "parte" em si não é de todo claro (VARZI, 2009). A pesquisa sobre as relações parte-todo tem sido orientada por três abordagens principais (GERSLT; PRIBBENOW, 1995): i) a abordagem lógica, usada para formalizar domínios e realizar modelagem ontológica (SMITH, 1993); ii) a abordagem psicológica, a qual estuda as assim chamadas "partonomias" no sistema conceitual humano (TVERSKY, 1990; TVERSKY, HEMENWAY, 1984); e iii) a abordagem linguística, que trata da abordagem semântica na linguagem natural (CRUSE, 1986; WINSTON; CHAFFIN; HERRMANN, 1987).

$\mathrm{Na}$ Ciência da Informação, as relações semânticas são usadas em Sistemas de Organização do Conhecimento (SOCs) para fins representação e posterior recuperação de informação. Neste contexto, o estudo das relações semânticas se fundamenta em abordagens Linguísticas e Psicológicas, como 
sugerido em trabalhos abrangentes da área, por exemplo, de Khoo e $\mathrm{Na}$ (2006) e de Hjorland (2007).

Este artigo objetiva proporcionar uma visão teórica bem fundamentada de noções de parte-todo para cientistas da informação. Trata-se de um suporte essencial para lidar com os diversos tipos de SOCs disponíveis, incluindo ontologias (SOUZA; TUDHOPE; ALMEIDA, 2012). A literatura da área não parece contemplar investigação de natureza teórica, combinando pontos de vista filosófico e linguístico. Além disso, pretende-se explicitar peculiaridades das relações parte-todo muitas vezes negligenciadas pela comunidade da Ciência da Informação.

Para tal, duas perspectivas sobre a noção de parte-todo são consideradas: a primeira perspectiva - de natureza não-formal, conhecida como relação meronímica - tem orientação psicolinguística e reconhece que relações semânticas entre unidades lexicais (por exemplo palavras, frases, etc.) são expressas linguisticamente de acordo com seu uso pela mente humana em vários contextos; a segunda perspectiva - de natureza formal, conhecida como relação mereológica - tem orientação lógico-filosófica e considera relações semânticas como uma questão de verdade analítica que carece de formalização.

Finalmente, emprega-se a propriedade da transitividade para distinguir as abordagens formal e não-formal. A transitividade é de longe a propriedade mais importante das relações parte-todo no contexto da recuperação da informação, a medida em que permite a expansão das consultas via cadeias transitivas (STOCK, 2010). Cabe citar que existem exemplos anteriores desse tipo de distinção em ontologias, por exemplo, Guizzardi (2009), Keet e Artale (2008). Discute-se as duas abordagens, sugerindo que a escolha por uma ou outra obedece a um critério pragmático no escopo do desenvolvimento de SOCs. A abordagem não-formal prevalece em tesauros, e a formal em ontologias.

O restante do presente artigo está organizado como segue: a seção 2 descreve as relações partitivas não formais - meronímias - e a seção 3 descreve as relações partitivas formais - mereologias; a seção 4 discute as diferenças entre estas duas relações, e a seção 5 conclui pela definição do tipo de relação usada em SOCs de acordo com a propriedade da transitividade. 


\section{RELAÇÕES PARTITIVAS NÃO-FORMAIS: MERONÍMIAS}

Do ponto de vista linguístico, dois aspectos sobre significado, diferentes ainda que relacionados, devem ser considerados na análise das relações. $O$ primeiro, denominado referência, trata das relações entre, por um lado as unidades lexicais, e por outro lado o mundo não linguístico da experiência. $O$ segundo aspecto, denominado sentido, refere-se ao complexo sistema de relações que se mantém entre os próprios elementos linguísticos, em particular, entre unidades lexicais (MORRIS, 2007).

Esse tipo de relação é muitas vezes nomeado pela expressão relações de sentido porque se acredita que tais relações ocorrem entre os sentidos das palavras (LYONS, 1977). A literatura proveniente da Linguística geralmente usa as expressões relação léxica ou relação semântica para se referir às relações paradigmáticas entre palavras (KHOO; NA, 2006).

Relações paradigmáticas conectam palavras que são membros de uma mesma categoria gramatical; relações sintagmáticas descrevem relações entre palavras de diferentes categorias gramaticais (MURPHY, 2003) (Quadro 1). Uma explicação abrangente sobre o assunto pode ser encontrada em Lyons (1968).

\begin{tabular}{c|c|c|c|c|c}
\multirow{2}{*}{ Relação } & \multicolumn{5}{c}{$\leftarrow$ Sintagmática $\rightarrow$} \\
$\begin{array}{c}\uparrow \\
\text { Paradigmática } \\
\downarrow\end{array}$ & Um & cão & pulou & no & tapete \\
\cline { 2 - 6 } & $\mathrm{O}$ & gato & sentou & na & esteira \\
\cline { 2 - 6 } & $\begin{array}{c}\text { Aquele } \\
\text { Fonte: adaptado de Murphy (2003). }\end{array}$ & $\begin{array}{c}\text { homem } \\
\text { comeu }\end{array}$ & de & chapéu \\
\end{tabular}

Nesta seção, enfatiza-se a relação léxica-semântica chamada meronímia. Meronímias são relações intra-linguísticas, cuja contrapartida formal se manifesta em relações mereológicas, por sua vez, extra-linguísticas. (Seção 3).

No restante da presente seção, apresenta-se uma visão geral das relações meronímicas (seção 2.1). Explica-se como essas relações são estabelecidas, como formam cadeias transitivas e, finalmente, como são diferenciadas de outras relações similares (seção 2.2). 


\subsection{RELAÇÕES MERONÍMICAS: UMA VISÃo GERAL}

A relação meronímica é um tipo de relação de parte-todo entre itens lexicais. Várias questões sobre meronímias vem sendo estudadas ao longo dos anos, por exemplo: i) a relação meronímica é uma única relação ou uma família de relações? ii) Se as meronímias são uma família de relações, como as relações podem ser diferenciadas de outras? iii) o que o termo "parte" realmente significa? iv) quais são os relata das relações meronímicas? O termo "relata", plural do termo latino "relatum", diz respeito as entidades participantes da relação, por exemplo, na sentença "João casa-se com Maria" os relata são "João" e "Maria". Não há consenso sobre as questões apresentadas, mas algumas considerações auxiliam no seu entendimento (LYONS, 1968).

Advoga-se que as meronímias não são uma relação única, mas sim um conjunto de várias relações mais ou menos similares entre si (CRUSE, 1986; WINSTON, CHAFFIN, HERRMANN, 1987). De fato, alguns autores defendem que, embora pareça fácil identificar diferentes tipos de relações meronímicas, os critérios usados para desenvolver tipologias dessas relações não diferenciam esses tipos (MURPHY, 2003).

Com relação ao significado real do termo "parte", trata-se realmente de um termo vago e genérico, usado para expressar uma variedade de relações semânticas distintas. Na segunda metade do século XX, o Roget's Thesaurus ${ }^{1}$ já listava cerca de 400 sinônimos para o termo "parte" e, havia ainda pelo menos 40 termos relacionados de escopo mais restrito (WINSTON, CHAFFIN, HERRMANN, 1987).

Com relação aos relata da relação meronímica, a abundância de termos representando entidades linguísticas - palavras, lexemas, itens lexicais, unidades lexicais, lemas, para mencionar apenas alguns -acaba por gerar dúvidas sobre o que as relações semânticas estão realmente relacionando. Os relata em uma meronímia são itens lexicais delimitados sintagmaticamente (MURPHY, 2003).

\footnotetext{
1 http://www.roget.org/
} 


\subsection{CARACTERIZAÇÃo dA RELAÇÃo MERONÍMICA}

Apresentam-se aqui características típicas das relações meronímicas, as formas como a relação se estabelece, a sua habilidade em formar cadeias, e sua distinção para outras relações similares.

\section{|Como identificar uma meronímia?}

No contexto de relações semânticas observa-se, intuitivamente, que algumas palavras parecem mais intimamente relacionadas em sentido do que outras. Alguns termos têm um escopo mais amplo de aplicação do que outros, por exemplo: "coisa" é mais amplo do que a "mobília", que por sua vez é mais amplo que a "mesa". Identifica-se aqui a relação semântica fundamental, à saber, a hiponímia, a qual se estabelece entre dois itens lexicais que mantém uma relação de classe-subclasse, por exemplo, os pares "maçã-fruta", "computadorequipamento" e "carro-veículo" (PRIBBENOW, 2002).

A meronímia é as vezes identificada pela comparação com a hiponímia, ou seja, como um tipo de relação hierárquica. A meronímia tem duas denominações, por exemplo: "braço-corpo" é uma meronímia; "corpo-braço" é uma holonímia (GERSLT; PRIBBENOW, 1995). A diferença entre uma meronímia e uma hiponímia é facilmente identificável quando as unidades lexicais envolvidas são substantivos representando objetos, por exemplo, "braço não é um corpo" (hiponímia), "braço é parte do corpo" (meronímia). Entretanto, existem diversas denominações, o que torna difícil identificar claramente apenas a meronímia apenas pelo nome (MURPHY, 2003):

- necessárias vs opcionais, por exemplo, respectivamente, "dedo-mão" e "lago-parque" (LYONS, 1977);

- canônica vs facultativa; por exemplo, respectivamente, "mão-braço" e "porta-maçaneta", já que nem toda porta tem maçaneta (CRUSE, 1986);

- intrínseca vs extrínseca; por exemplo, respectivamente, "mão-braço" e "porta-maçaneta" (CROFT; CRUSE, 2004);

- componente-objeto, membro-coleção, porção-massa, objeto-material, característica-atividade, local-área, por exemplo: "pedal-bicicleta", "cão- 
matilha", "fatia-bolo", "roda-carro", "pagar-comprar", "Curitiba-Paraná" (WINSTON, CHAFFIN, HERRMANN, 1987);

- membro-coleção, todo-funcionário, organização-liderança, todo-centro todo-unidade, por exemplo, "carro-frota", "aeronave-tripulação", "floconeve", "navio-capitão", "montanha-pico" (WANNER, 1996).

Como pode ser verificado, em alguns exemplos os mesmos tipos de relações são definidas por termos diferentes. Dessa forma, mais detalhes sobre como caracterizar a relação meronímica são necessários.

\section{|Como a relação de meronímia se estabelece?}

Dois tipos de testes básicos podem ser usados para verificar como se estabelece a meronímia entre unidades lexicais. O primeiro teste considera uma sentença na forma "um $Y$ tem $X s$ (ou $X$ )", por exemplo, "um livro tem páginas", onde $Y=$ "livro" e "Xs= "páginas". O segundo teste considera uma sentença na forma "um $X$ é parte de um $Y$ ", por exemplo, "uma conta bancária com muito dinheiro é parte da atratividade de um homem", onde X="conta bancária com muito dinheiro" e $\mathrm{Y}=$ "atratividade de um homem". Uma vez atendidos esses dois testes, teoricamente, considera-se a existência de uma meronímia (CRUSE, 1986).

Entretanto, meronímias também podem se estabelecer a partir de significados interpretados contextualmente. Nesse caso, a validação de relações baseia-se em testes considerando a sentença "se A é meronímia de $B$, em um contexto específico, qualquer membro de $A$ deve atender a uma das seguintes condições": i) ou A é mapeado para um membro de B do qual se interpreta que ele é parte; ii) ou A permanece numa relação de parte-todo intrinsecamente interpretada com algum membro de B potencial ou real, por exemplo, "o nariz é intrinsecamente parte do rosto" (CROFT; CRUSE 2004).

Além disso, um conjunto de outros fatores pode ser considerado para identificar o estabelecimento de uma relação meronímica de " $X$ como parte de Y" (CROFT; CRUSE, 2004): i) o limite de $X$ não transgrida o limite de $Y$; ii) $X$ compartilha todas as suas substâncias com $Y$; iii) os limites de $\mathrm{X}$ podem ser 
identificados no todo $Y$; iv) quanto mais saliente a descontinuidade entre $X$ e não$X$, mais evidente é a parte; v) quanto maior a coesão interna de $X$, mais evidente é a parte; vi) $X$ tem uma função definida em relação a $Y$, por exemplo, "asa serve para voar"; vii) $X$ é autônomo, isto é, uma réplica exata de $X$ também conta como parte; e viii) existe consistência de tipos entre $\mathrm{X}$ e $\mathrm{Y}$, por exemplo, partes de objetos são objetos, parte de processos são processos.

\section{Cadeias meronímicas}

Meronímias são caracterizados ainda pela sua habilidade em gerar cadeias de elementos conectados. Transitividade é uma propriedade de relações semânticas que pode ser expressa de forma simples pela regra: "se $x$ é parte de y e y é parte de $z$, então $x$ é parte de z". Por exemplo, "se João mora com Maria e Maria mora com Pedro, então João mora com Pedro". A relação de meronímia é, em geral, considerada transitiva, mas essa consideração não é consensual, uma vez que são apresentados casos em que a propriedade teoricamente não se mantém (BITTNER, 2011).

Uma cadeia meronímica básica tem uma parte mínima que em si não tem outras partes, além de um todo que é o todo máximo (BIERWISCH; HEIDOLPH, 1970). Por exemplo, "a ponta do dedo é parte do dedo, o dedo é parte da mão, a mão é parte do braço, o braço é parte do corpo". Decidir limites máximo e mínimo depende de detalhes nem sempre claros, mas existem caracterizações que auxiliam a identificar o início e o término da cadeia, à saber: o escopo de predicação das partes, e a noção de consistência de tipos (CROFT; CRUSE, 2004).

A questão do escopo de predicação das partes se baseia na premissa de que um elemento $A$, constituinte de uma certa cadeia, é parte-imediata de outro elemento B; por sua vez, B é um todo-imediato de A. O todo-imediato é o escopo normal de predicação para as partes, o qual, uma vez atendido, vai gerar uma sentença aceitável. Por exemplo, uma sentença normal e aceitável é "o dedo é parte-imediata da mão, enquanto a mão é todo-imediato do conjunto de dedos". No conjunto de sentenças "um corpo tem dois braços, uma mão tem cinco dedos, 
um dedo tem três junções de dedo e uma unha, um braço tem cinco dedos, um corpo tem vinte e oito junções de dedos", enquanto as três primeiras sentenças são aceitáveis, as duas últimas não são (CROFT; CRUSE, 2004).

Outro aspecto relevante é a noção de consistência de tipos que distingue dois tipos principais de partes, denominadas "partes-segmentais" e "partessistêmicas" (CRUSE, 1986). Partes-segmentais são delimitadas, e estão em sequência quando o todo é perpassado espacialmente, por exemplo: as partes visíveis do corpo, tais como braços, pernas, cabeça, etc. Partes-sistêmicas não são perpassáveis do ponto de vista espacial, são funcionalmente distintas e possuem consistência interna, por exemplo: sistema nervoso, o esqueleto, etc.

\section{|Relações similares às meronímias}

A mera observação de uma situação nem sempre permite estabelecer as relações presentes com precisão. Podem ocorrer erros na identificação de relações, e mesmo erros na diferenciação entre meronímias e outras relações semânticas similares. Uma questão, nesse contexto, é considerar se certas relações são variações de meronímias ou relações de natureza distinta (MURPHY, 2003). Exemplos de relações semelhantes às meronímias são: i) posse, por exemplo, "milionário-dinheiro"; ii) atribuição, por exemplo, "mansãogrande"; iii) inclusão locativa, por exemplo, "a mulher está na sala, a sala está na casa, a casa está na cidade" (MILLER; JOHNSON-LAIRD, 1976).

As diferenças e similaridades entre duas relações específicas - parteporção e parte- fragmento - contribuem para identificação de distinções entre as meronímias e relações similares. De fato, a meronímia é uma variedade de uma relação mais genérica denominada porção-todo. A noção básica de "porção" é o fato de uma região espacial conter outra, por exemplo: "uma porção do bolo foi dada a cada convidado", "minha porção da omelete tinha pedaços de casca de ovo", etc. Uma "parte" é uma hiponímia de "porção" (CROFT; CRUSE, 2004). Da mesma forma, "fragmento" também é hiponímia de "porção", a qual, entretanto, não se qualifica como "parte-todo", uma vez que fragmentos não mantém relações com seus todos. Por exemplo, usando uma serra para cortar uma 
computador em porções arbitrárias obtém-se fragmentos, mas não partes do computador. Para obter partes do computador, é necessário usar ferramentas para desmontá-lo, separando as partes. "Fragmentos" são diferenciados de "partes" porque não são contemporâneos de seus todos, ou seja, não há fragmentos até que o todo seja destruído (CRUSE, 1986).

As "partes" e os "fragmentos" em comum a seguintes características: estabilidade topológica e continuidade espacial. Por outro lado, "partes" são distintas de "fragmentos" por outras: autonomia, limites não arbitrários e função determinada (CROFT; CRUSE, 2004).

Um contra exemplo para a noção de estabilidade topológica é "não existe algo que possa ter um fragmento ou ter uma parte do vapor". A continuidade espacial pressupõe que o movimento de qualquer ponto dentro de um "fragmento" não atravessa o material não pertencente àquele fragmento. A continuidade espacial funciona da mesma forma para "partes". Para autonomia, considere-se que um "fragmento" do computador pode ser novamente integrado a ele, mas a autonomia não funciona para "partes": partes de um computador não precisam pertencer ao computador original. Assim, "partes" são autônomas, "fragmentos" não são. Os limites não arbitrários delimitam delimitadas por uma descontinuidade, por exemplo, o antebraço delimitado por cotovelo e punho. Assim, é possível apontar "partes" de um todo via limites não arbitrários, mas não há como se referir a "fragmentos" de um todo. A característica função determinada sugere que as partes têm funções relativas aos respectivos todos, por exemplo, "olho para ver", e "freio para parar" (CROFT; CRUSE, 2004).

\section{RELAÇÕES PARTITIVAS FORMAIS: MEREOLOGIAS}

Conforme já mencionado, a relação de meronímia abordada na seção anterior (seção 2), é uma relação intra-linguística entre unidades lexicais. A contrapartida formal da meronímia é a relação mereológica. Portanto, ao contrário da meronímia, a relação mereológica é extra-linguística.

O estudo das relações parte-todo remonta à antiga Filosofia grega, quando foi investigada no âmbito de uma disciplina denominada mereologia (da 
palavra grega "meros" que significa "parte"). Em tempos modernos, no início do século XX, o principal destaque dessa teoria é o trabalho de Edmund Husserl². As teorias mereológicas mais aceitas, no entanto são posteriores e foram apresentadas por Stanisław Leśniewski ${ }^{3}$ em 1916 e por Henry Leonard ${ }^{4}$ e Nelson Goodman ${ }^{5}$ em meados do século XX.

Nesta seção, depois de apresentar uma visão geral das relações mereológicas (seção 3.1), descrevem-se brevemente as teorias mereológicas mais conhecidas, à saber: mereologia básica, a mereologia extensional e a mereologia clássica (seção 3.2). A versão clássica corresponde aos sistemas devidos a Leśniewski e a Leonard e Goodman, dos quais uma visão simplificada é apresentada.

\subsection{RELAÇõES MEREOLÓGICAS: UMA VISÃO GERAL}

O termo mereologia dá nome a teoria que lida com as relações de partes com um todo e as relações de partes para partes dentro de um todo, do ponto de vista formal. Atualmente, o termo mereologia ainda é usado para se referir ao estudo formal de um todo e suas partes em Lógica e Filosofia (SMITH; KUNNE, 2004)

Existem partes de objetos, partes de eventos, de entidades abstratas, de entidades temporais ou espaciais, dentre outras, por exemplo (SIMONS, 1987): "homem-cabeça", "árvore-tronco", "casa-telhado", "montanha-cume", "batalhatiro", "vida de inseto-estágio larval", "romance-primeiro capítulo", etc.

No entanto, uma observação atenta mostra que o significado exato do termo "parte" não é tão claro, considerando a multiplicidade de situações em que é empregado. Exemplos dessa multiplicidade são (VARZI, 2009): "a alça faz parte da caneca", em que a parte é conectada ao todo; "a tampa é parte da minha caneta, em que a parte é desconectada do todo; "a metade esquerda é a sua parte do bolo", onde a parte é arbitrariamente demarcada; "os talheres fazem

\footnotetext{
2 Edmund Gustav Albrecht Husserl: filósofo alemão (1859 - 1938).

${ }^{3}$ Stanisław Leśniewski: filósofo e lógico polonês (1886 - 1939).

${ }^{4}$ Henry S. Leonard: filósofo norte-americano (??? - ???).

${ }^{5}$ Henry Nelson Goodman: filósofo norte-americano (1906 - 1998).
} 
parte do faqueiro" é um exemplo de parte desconectada do todo; "o conteúdo desta bolsa é parte do que eu comprei" é um exemplo de partes materiais; "essa área é parte da sala de estar" é um exemplo de partes imateriais; "o primeiro ato foi a melhor parte da peça" é um exemplo de parte temporal.

Dois grupos de princípios, acrescidos de algumas noções básicas, dão origem ao núcleo das teorias mereológicas utilizadas para explicar as relações entre partes e todos: i) princípios de decomposição, que partem de um todo para suas partes; e ii) princípios de composição, que partem de partes para um todo. No escopo desses princípios, surgem e evoluem as teorias mereológicas.

\subsection{TEORIAS DA MEREOLOGIA}

Existem princípios básicos para sua identificação da mereologia. A partir desses princípios, é possível alcançar teorias mais sofisticadas capazes de explicar a diversidade de noções existentes. Essas teorias são apresentadas no restante dessa seção.

\section{|Mereologia básica}

Ainda que seja necessário um certo nível de formalização para explicar as teorias da mereologia, não se apresentam aqui sentenças formais em representação lógica, mas sim elucidações em linguagem natural. Essa decisão parte premissa que as formalizações não são totalmente necessárias para os objetivos do presente artigo, e do fato de que a pesquisa e a formação em Ciência da Informação nem sempre contemplam Lógica usada em Filosofia.

Inicialmente, considerando que o predicado de uma sentença é definido como uma relação parte-todo e, que o termo "parte" significa o tipo de relação (exemplificado na seção 3.1), introduz-se a noção de ordenamento parcial (BITTNER, 2011). Um ordenamento parcial é uma relação binária, ou seja, uma relação entre elementos de dois conjuntos, que tem como propriedades a reflexividade, transitividade e assimetria (Quadro 2). 


\section{Quadro 2 - Caracterizações mereológicas básicas}

\begin{tabular}{l|l|l|l} 
Ref. & Propriedade & \multicolumn{1}{|c|}{$\begin{array}{c}\text { Formulação em } \\
\text { linguagem natural }\end{array}$} & \multicolumn{1}{c}{ Exemplo } \\
\hline$(1)$ & Reflexividade & x é parte de $x$ & $\begin{array}{l}\text { João é parecido consigo } \\
\text { mesmo. }\end{array}$ \\
\hline$(2)$ & Transitividade & $\begin{array}{l}\text { Se } x \text { é parte de y e y é parte } \\
\text { de } z \text {, então } x \text { é parte de } z .\end{array}$ & $\begin{array}{l}\text { João mora com Pedro e Pedro } \\
\text { mora com Maria, João mora } \\
\text { com Maria. }\end{array}$ \\
\hline (3) & Simetria & $\begin{array}{l}\text { Se } x \text { é parte de y e y é parte } \\
\text { de } z, \text { então } x \text { é igual a } z .\end{array}$ & $\begin{array}{l}\text { Se João é casado com Maria, } \\
\text { Maria é casada com João. }\end{array}$
\end{tabular}

Fonte: elaborado pelos autores, 2019.

Nos exemplos, as sentenças capturam intuições que as pessoas naturalmente têm envolvendo relação parte-todo e as propriedades recémmencionadas. A reflexividade significa que tudo é parte de si mesmo; a transitividade significa que qualquer parte de outra parte de uma coisa é também parte daquela coisa; e a assimetria significa que duas coisas distintas não podem ser parte uma da outra.

A teoria incorporada no Quadro 2, conhecida como Mereologia Básica, é a base comum para qualquer teoria parte-todo. À essa fundamentação inicial composta pelas propriedades básicas (Quadro 2), adicionam-se outras propriedades, à saber: igualdade, parte-própria, sobreposição superior e inferior 6 (Quadro 3). Como não é importante para propósitos desse artigo, omite-se a explicação de como as sentenças de (4) a (7) são obtidas a partir de (1) a (3).

\section{Quadro 3 - Caracterizações mereológicas adicionais}

\begin{tabular}{l|l|l} 
Ref. & Propriedade & \multicolumn{1}{c}{ Formulação em linguagem natural } \\
\hline$(4)$ & Igualdade & $\begin{array}{l}\text { x é igual a y corresponde a situação na qual x é parte de y } \\
\text { e y é parte de x. }\end{array}$ \\
\hline$(5)$ & Parte própria & $\begin{array}{l}\text { x é parte própria de y corresponde a situação na qual x é } \\
\text { parte de y e y não é parte de x. }\end{array}$ \\
\hline$(6)$ & $\begin{array}{l}\text { Sobreposição } \\
\text { superior }\end{array}$ & $\begin{array}{l}\text { x sobrepõe-se (superior) a y corresponde a situação em } \\
\text { que há um z tal que z é parte de x e z é parte de y. }\end{array}$ \\
\hline (7) & $\begin{array}{l}\text { Sobreposição } \\
\text { inferior }\end{array}$ & $\begin{array}{l}\text { x sobrepõe-se (inferior) de y corresponde a situação na } \\
\text { qual há um z tal que x é parte de z e y é parte de } z .\end{array}$
\end{tabular}

Fonte: elaborado pelos autores, 2019.

\footnotetext{
${ }^{6}$ Tradução livre dos termos em lingua inglesa "overlapping" e "underlapping".
} 
Para maior clareza, apresenta-se a forma gráfica das noções correspondentes (Figura 1).

Figura 1 - Propriedades aplicadas a relação partitiva entre x e y

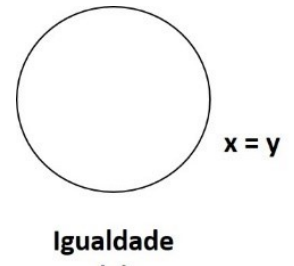

(a)

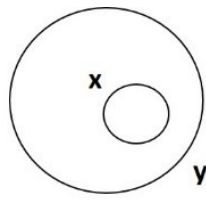

Parte própria

(b)

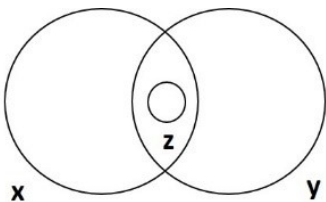

Sobreposição superior

(c)

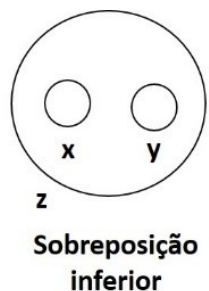

(d)

Fonte: elaborado pelos autores, 2019.

"Igualdade" significa que, se dois objetos são ao mesmo tempo parte um do outro, são na verdade o mesmo objeto e assim representados por uma única linha (Figura 1a). "Parte-própria" significa que x é parte de y, mas não é igual a y (Figura 1b), por exemplo, a "mão é parte da pessoa, mas não é (e nunca será) a pessoa como um todo". "Sobreposição" (superior), intuitivamente, significa que, se duas coisas x e y ocupam parcialmente o mesmo espaço, existe uma coisa $z$ que é parte de ambas outras duas coisas (x e y) (Figura 1c). Por exemplo, duas estradas cruzadas se sobrepõem onde há um entroncamento. Por outro lado, "sobreposição" (inferior) diz respeito a duas coisas x e y que são parte de outra coisa maior z (Figura 1d). Por exemplo, o polegar esquerdo e o dedo indicador se sobrepõem pois são partes da pessoa.

Cabe destacar que a Figura 1 apresenta apenas algumas possibilidades mais intuitivas para simplicidade. Uma visão completa das possibilidades está disponível em Varzi (1996).

\section{|Mereologia extensional}

A primeira extensão à mereologia básica é chamada de Mereologia Extensional, e envolve princípios de decomposição, ou seja, princípios que partem do todo em direção as partes. A noção central de decomposição considera que se algo tem uma parte própria, na verdade tem mais de uma. Em outras palavras, nada pode ter uma única parte própria. Isso implica na 
existência de um "resto" entre um todo e sua parte-própria, em qualquer processo de decomposição, denominado diferença mereológica.

Há mais de uma possibilidade de capturar formalmente a intuição da diferença mereológica. A possibilidade mais aceita é denominada suplementação (Quadro 4). De acordo com o princípio da suplementação, toda parte própria de um todo é suplementada por outra parte, que é disjunta da primeira. Essa última caracterização - a disjunção - captura a noção de diferença mereológica. Existe uma versão ligeiramente diferente do princípio da suplementação, a suplementação forte (Quadro 4), a qual corresponde à ideia de que, se um objeto não permite incluir outro entre suas partes, então deve haver um resto.

\begin{tabular}{c|l|l}
\multicolumn{3}{|c}{ Quadro 4 - Caracterizações extensionais - suplementação } \\
Ref. & Propriedade & \multicolumn{1}{c}{ Explicação em linguagem natural } \\
\hline (8) & Suplementação & $\begin{array}{l}\text { Se x é uma parte própria de y, então existe um z tal } \\
\text { que z é parte de y e z não sobrepõe x. }\end{array}$ \\
\hline (9) & $\begin{array}{l}\text { Suplementação } \\
\text { forte }\end{array}$ & $\begin{array}{l}\text { Se } x \text { não é parte de y, então existe um z tal que z é } \\
\text { parte de y e z não sobrepõe x. }\end{array}$
\end{tabular}

Fonte: elaborado pelos autores, 2019.

Ao adicionar o princípio da suplementação forte à mereologia básica, obtém-se a Mereologia Extensional. No âmbito desta, revela-se uma propriedade relevante, denominada extensionalidade. Para os propósitos desse artigo, é suficiente dizer que a extensionalidade assegura que as entidades são completamente definidas por suas partes.

\section{|Mereologia clássica}

A segunda extensão à mereologia básica, denominada Mereologia Clássica, envolve princípios de composição, ou seja, princípios que levam das partes em direção ao todo. A noção da composição considera que sempre que existem coisas, existe também um todo que é formado exatamente por essas coisas. Em outras palavras, existe sempre uma soma única para entidades arbitrárias. A singularidade da soma é garantida pela propriedade da extensionalidade, implícita no princípio da suplementação no âmbito da 
mereologia extensional (seção 3.2.2).

A existência dessa soma implica que sempre há uma fusão entre duas ou mais partes, a qual é conhecida como soma mereológica. De forma semelhante ao caso da mereologia extensional, existe mais de uma possibilidade para capturar a noção da soma mereológica. A opção mais fraca é denominada limite superior e uma opção menos fraca é denominada soma (Quadro 5).

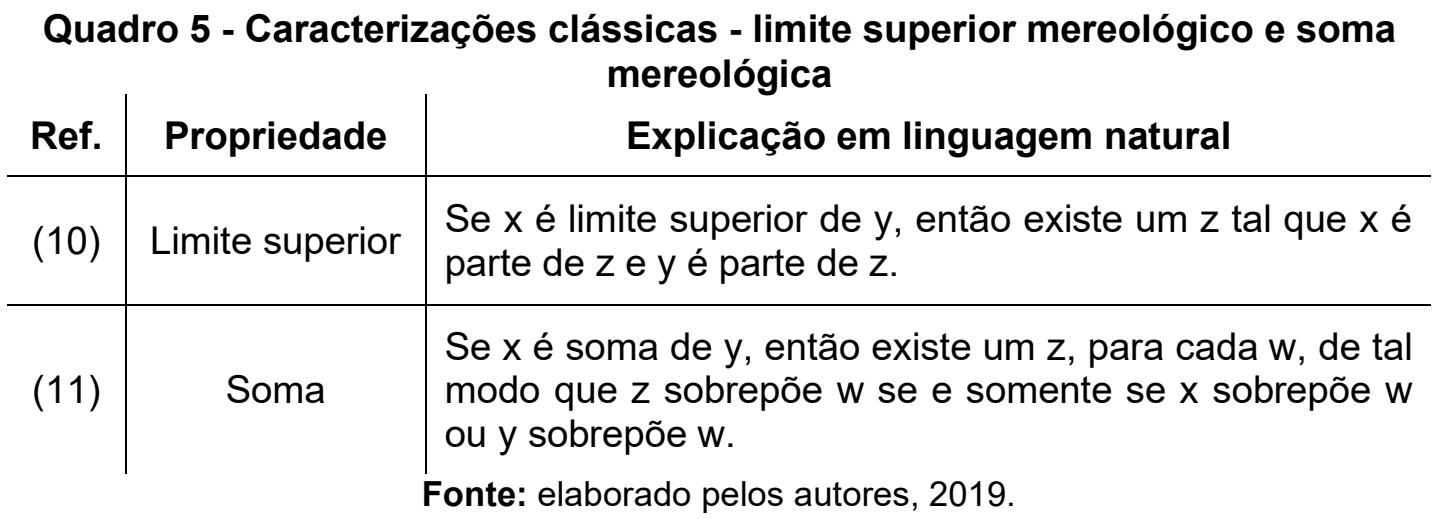

Um limite superior mereológico de dois objetos é, de fato, um outro objeto, do qual ambos os originais são parte. A soma mereológica é um limite superior mereológico no qual qualquer parte se sobrepõe a um dos indivíduos somados (GOTTS; COHN, 1995). Em outras palavras, uma soma mereológica entre dois objetos é algo composto exatamente de suas partes e nada mais.

As formulações acima mencionadas tentam capturar as noções de uma soma de objetos que formam um todo. No entanto, elas não são suficientes pois para acesso à mereologia clássica, a formulação deve considerar limites infinitivos e somas infinitivas. Esses elementos infinitivos exigem o uso de uma lógica infinitiva ${ }^{7}$, que lida com operações infinitivas, isto é, operações que podem ter um número infinito de valores de entrada para produzir uma saída (BELL, 2012).

Usando essas ferramentas, pode-se expressar a versão mais forte dos princípios da composição, ou seja, a composição irrestrita que permite somas arbitrárias. O ferramental necessário para entender este último tipo de composição não é apresentado aqui pela dificuldade inerente em explicá-lo em

\footnotetext{
7 https://pt.wikipedia.org/wiki/Lógica_infinitária.
} 
apenas um artigo. Ao realizar essa tarefa, é possível alcançar a Mereologia Clássica devida a Leśniewski (1992) e Leonard e Goodman (1940).

\section{DISCUSSÃO: MERONÍMIA OU MEREOLOGIA?}

Até aqui, foram apresentados estudos originados principalmente na Lógica e na Linguística, para lidar com a multiplicidade de aspectos que envolvem a noção de parte-todo. No restante desta seção, discute-se a questão da transitividade das relações parte-todo, como aspecto central para a diferenciação entre os dois tipos de relação, à saber, meronímica e mereológica.

A transitividade é uma propriedade das relações semânticas de suma importância no contexto da representação da informação e do conhecimento, por pelo menos duas razões principais: i) não há consenso se transitividade é válida em todas as relações parte-todo; ii) a transitividade entre termos que denotam entidades permite a expansão de consultas ao longo de uma cadeia transitiva, uma característica essencial para os SOCs (WELLER, STOCK, 2008).

Na seção seguinte (seção 4.1), discute-se a transitividade das meronímias observando que as iniciativas linguísticas muitas vezes são tentativas de fornecer respostas para casos em que meronímias não parecem transitivas. $\mathrm{Na}$ seção posterior (seção 4.2), apresentam-se argumentos a favor da transitividade das relações parte-todo, como proposto pela mereologia.

\subsection{TRANSITIVIDADE DAS RELAÇÕES MERONÍMICAS}

No âmbito da Linguística, existem abordagens sugerindo que alguns sentidos legítimos do termo "parte" não admitem transitividade. Outro autores não concordam com a suposta falha na transitividade e, mesmo, apresentam casos diferenciados: i) as relações meronímicas são transitivas (LYONS, 1977; CRUSE, 1986); ii) as falhas na transitividade meronímica são ilusórias (CROFT; CRUSE, 2004); iii) ao invés de uma única relação, a meronímia é uma família de relações e a aparente não-transitividade envolve, na verdade, diferentes tipos de relações (WINSTON, CHAFFIN, HERRMANN, 1987); iv) as meronímias representam um grupo de relações, algumas das quais são transitivas, outras 
não (CHAFFIN, HERRMANN, WINSTON, 1988).

Lyons (1977) enfatiza a diferença entre, de um lado, as entidades do mundo - partes separáveis de uma coisa e o todo do qual são partes - e, de outro lado, o vocabulário empregado para se referir a essas entidades. Considerando tal diferença, a transitividade é mantida entre entidades físicas, mas não vale para todas as relações lexicais usadas para se referir a essas entidades. Por exemplo, "o dedo do professor é parte do professor, o professor é parte da universidade, então o dedo do professor é parte da universidade" mostra uma sentença inaceitável (a terceira), que põe em dúvida a transitividade das meronímias.

A possibilidade de identificar relações meronímicas transitivas e não transitivas pode evidenciar a existência de mais de um tipo de meronímia.

Cruse (1986) atribui falhas de transitividade nas meronímias a dois aspectos: a noção de domínio funcional, e a diferença entre dois tipos de partes, à saber, os anexos e as partes integrais. Por exemplo:

(1a) O casaco tem mangas.

(1b) As mangas tem punhos.

(1c) O casaco tem punhos. (2a) A casa tem uma porta.

(2b) A porta tem uma maçaneta.

(2c) A casa tem uma maçaneta.

No primeiro conjunto de sentenças, (1c) é uma conclusão válida de (1a) e (1b). Considerando o segundo conjunto de sentenças, não se pode dizer que (2c) é uma conclusão válida de (2a) e (2b). Isso ocorre porque a parte tem uma "função" em relação ao todo. Assim, no segundo exemplo, o domínio funcional da maçaneta é restrito: uma maçaneta serve para abrir uma porta, mas não tem função com relação a algo maior (do qual a porta faz parte) como uma casa. Ao contrário, no primeiro exemplo, o domínio funcional de "punhos da camisa" é generalizado: a função dos punhos é decorativa, para mangas e jaquetas. Assim, se o contexto do domínio funcional é generalizado, as meronímias são transitivas; se domínio funcional é restrito, as meronímias são não-transitivas.

Para entender a questão dos anexos versus partes integrais é preciso diferenciá-los. A integridade de uma entidade é destruída se faltar uma parte integral, mas isso não é verdade se a parte ausente for um anexo. Por exemplo, é normal fazer referência aos anexos como conectados a um objeto maior, como 
na sentença "a placa está anexada à porta" porque a porta não deixa de existir se faltar a placa; mas é estranho referir-se a partes integrais da mesma forma, como na frase "o cabo é anexado à colher", porque não há colher se não houver cabo. Nessa visão, meronímias são transitivas apenas quando os relata da relação são partes integrais.

Croft e Cruse (2004) partem dos exemplos fornecidos por Cruse (1986) para sugerir que falhas na transitividade meronímica são ilusórias. Winston, Chaffin, Herrmann (1987) corrobora com essa ideia, explicando que as meronímias parecem não ser transitivas quando ocorre uma mistura de mais de um tipo de relação. Por exemplo:

(2a) A geladeira é parte da cozinha.

(2b) A cozinha é parte da casa.

(2c) A geladeira é parte da casa.

Em (2c) há uma falha de transitividade devida ao uso de uma relação objeto-componente em (2a) junto a uma relação de local-área em (2b). Winston, Chaffin, Herrmann (1987) concluem que a relação meronímica é transitiva desde que se mantenha um único sentido para o termo "parte" em todas as situações. Caso contrário, como no caso de relações objeto-componente ou local-área, ocorrem falhas de transitividade.

\subsection{TRANSITIVIDADE DAS RELAÇÕES MEREOLÓGICAS}

Como apresentado até aqui, existem propostas que sugerem que a relação de meronímia é não-transitiva (seção 4.1). No entanto, existem também propostas sugerindo que essa questão da não-transitividade não é legítima (SIMONS, 1987; VARZI, 2006; JOHANSSON, 2004; KEET; ARTALE, 2008).

Alguns exemplos, já citados nesse artigo, são casos clássicos usados para sugerir a não transitividade do parte-todo: "a maçaneta é parte da porta e a porta é parte da casa, mas a maçaneta não é parte da casa" (CRUSE, 1979). Outros exemplos são: "o núcleo é parte da célula e a célula é parte do órgão, mas o núcleo não é parte do órgão"; "o pelotão faz parte de uma divisão, uma divisão faz parte de um batalhão, mas um pelotão não faz parte de um batalhão" (RESCHER, 1955). 
Simons (1987) corrobora com Winston, Chaffin, Herrmann (1987) ao explicar que a falha na transitividade surge quando se consideram sentidos extrínsecos à teoria parte-todo. Por exemplo, quando se considera uma função, em ambos os casos de "maçaneta-casa" e "núcleo-célula". No caso de "batalhãopelotão, o sentido que não é transitivo tem a ver com linhas de comando no âmbito de uma instituição (o exército). Considerando apenas aspectos intrínsecos, a relação parte-todo é transitiva.

Com relação ao exemplo clássico de não-transitividade, o caso acima mencionado "maçaneta-casa", Varzi (2006) afirma que, a aparente nãotransitividade teria a ver com a existência de um modificador de predicado "invisível", representado aqui por $\phi$. A relação parte-todo é transitiva, mas as relações " $\phi$-parte-todo" não são necessariamente, exatamente porque não representam a relação genuína entre partes. Por exemplo, em "o núcleo é parte da célula e a célula é parte do órgão, mas o núcleo não é parte do órgão" o núcleo não é uma parte distinta do órgão, mas o núcleo é uma parte distinta da célula, bem como a célula é uma parte distinta do órgão.

Johansson (2004) completa o entendimento explicando que as relações parte-todo são transitivas na Mereologia porque, nesse contexto, as partes podem ser apenas espaciais ou temporais. Johansson (2004), além de aceitar o modificador de predicado $\phi$ sugerido por Varzi (2006), o qual modifica o significado original da relação parte-todo, revela sua natureza: a aridade das relações. A aridade de uma relação é o número de elementos envolvidos em qualquer instância dessa relação: se há dois elementos envolvidos, como x e y, então a relação entre eles é binária; se houver três elementos envolvidos, como $x, y$ e z, a relação é ternária; e assim por diante. As relações parte-todo mereológicas são relações binárias e transitivas; meronímias e outras relações similares - como a relação mereológica mais um modificador de predicado $\phi-$ nem sempre são binários ou transitivos.

Um exemplo simples é a relação na sentença "x é-tia de y". Apesar de parecer binária, existe outra entidade $z$ tal que "x é-irmão-de z" e "z é-pai de y". Em situações desse tipo, assim como para outros valores do modificador $\phi$, a composição $\phi$-parte não é binária, e sim uma relação na qual pelo menos três 
relata estão envolvidos. Nesses casos, as propriedades mereológicas, como a transitividade, não podem ser sempre consideradas.

Keet e Artale (2008) propõem uma taxonomia que distingue relações meronímicas e mereológicas em um único esquema. As diferenças entre as relações parte-todo são atribuídas aos diferentes tipos de entidades utilizadas como relata das relações. Além disso, o ramo da taxonomia denominado "é simbolizado por " $m$-parte-de", no qual a letra " $m$ " parece referir-se a alguma variação dos modificadores sugeridos por Varzi (2006) e Johansson (2004).

\section{CONSIDERAÇÕES FINAIS}

O presente artigo apresentou as duas principais modalidades de relação parte-todo, à saber: a meronímia, caracterizada como relação intra-linguística não formal; a mereologia, caracterizada como relação extra-linguística formal. Após caracterizar esses dois tipos de relações envolvidos na noção de partetodo, apresentou-se a propriedade da transitividade como forma de diferenciar essas relações. Finalmente, cabe considerar como tal diferenciação é útil no escopo de concepção e uso de SOCs, em particular, tesauros e ontologias.

Em relação a transitividade das relações parte-todo, de um lado, filósofos interessados na mereologia mantém a validade da transitividade sempre que o termo "parte-todo" conservar seu sentido original, ou seja: i) quando os relata são entidades substanciais e entidades processuais (SMITH; MUNN, 2008); ii) quando os relata envolvidos são espaciais ou temporais (JOHANSSON, 2004); iii) quando a relação é independente de domínio (VARZI, 2006). Por outro lado, a Linguística revela situações em que as relações meronímicas, não mantêm a transitividade: i) nem todas relações lexicais mantém-se transitivas (LYONS, 1977); ii) a transitividade é dependente do contexto (CROFT; CRUSE, 2004); iii) transitividade depende do sentido atribuído a "parte" (WINSTON, CHAFFIN, HERRMANN, 1987).

Assim, sugere-se aqui o que parece ser claro em questões pragmáticas no escopo da construção de SOCs. Para estabelecer relações para fins de recuperação da informação, parece razoável considerar a riqueza da linguagem 
natural, considerando ainda que os documentos a recuperar são escritos usando tais linguagens. Entretanto, do ponto de vista teórico, seria interessante ao cientista da informação conhecer a teoria básica, válida em um contexto espaçotemporal (mereologia), para depois enfrentar as complexidades e amplitude das relações que envolve unidades lexicais (meronímia).

No desenvolvimento de tesauros, não há necessidade obrigatória de alto rigor na definição das relações. Pode-se empregar a relação termo-genérico e a relação termo-específico para definir a taxonomia, além da relação termorelacionado para representar o parte-todo. Essas relações são geralmente encontradas em tesauros, por exemplo, o Medical Subject Headings (MeSH) ${ }^{8}$, um vocabulário controlado criado pela National Library of Medicine dos EUA, para indexar, catalogar e pesquisar dados biomédicos. Exemplos de relações que se pode encontrar no MeSH são: "sangue-fetal termo-específico sangue", e "plasma termo-especifico sangue" (SCHULZ et al., 2009).

Do ponto de vista da recuperação da informação, essas relações atendem às necessidades de indexação e recuperação da informação em documentos, pois trabalhos científicos sobre "plasma sanguíneo" serão recuperados a partir de uma consulta sobre "sangue", assim como os artigos sobre "sangue fetal". No entanto, do ponto de vista ontológico, este exemplo contém duas relações distintas: "plasma parte-de sangue" denota uma relação parte-todo, e "sanguefetal é-um sangue" denota uma relação de classe-subclasse. Então, se o SOC em construção é uma ontologia, há necessidade de maior precisão na definição de relações, na medida em que ontologias são instrumentos criados para uso por computadores.

Em última análise, a definição de uma interpretação clara para o tipo de relação, bem como de propriedades como a transitividade, é particularmente importante quando se busca automatizar a expansão de consultas. Cabe ainda citar exemplos de questões e restrições que envolvem a escolha da mereologia na construção de ontologias, as quais são muitas vezes negligenciadas. A propriedade de extensionalidade da mereologia extensional afirma que

\footnotetext{
${ }^{8}$ https://www.nlm.nih.gov/mesh/meshhome.html
} 
entidades são definidas por suas partes. Assim, as palavras do inglês "on" e "no" seriam as mesmas, pois ambas são compostas pelas mesmas partes, a saber "o" e "n" (PRIBBENOW, 2002). Da mesma forma, a mereologia clássica garante que se possa somar objetos arbitrários para alcançar um todo, permitindo assim somar "meu estômago", "meu carro" e "o canto mais quente do meu gabinete" (JANSEN, 2008). Essas situações, bem como outras, claramente indesejáveis, deve receber atenção na concepção do modelo. Uma listagem desses problemas em ontologias será objeto de trabalhos futuros.

Acredita-se que a ontologia como disciplina tem muitos pontos de contato com as teorias tradicionais da Ciência da Informação, ao tratar, por exemplo, de assuntos seminais como classificação, tornando-se assim um tema genuíno e frutífero para a Ciência da Informação. Pretende-te continuar explorando esse tema em trabalhos futuros.

\section{REFERÊNCIAS}

BELL, J. L. Infinitary Logic. 2012. Disponível em:http://plato.stanford.edu/entries/logic-infinitary/. Acesso em: 23 jan. 2013.

BITTNER, T. Mereology 2. 2011. Disponível em:http://ontology.buffalo.edu/smith/courses03/tb/Mereology2.pdf. Acesso em: 15 ago. 2010.

BIEDERMAN, I.A Recognition-by-Components: a theory of human image understanding. Psychological Review, v. 94, p.115-147, 1987.

BIERWISCH, M.; HEIDOLPH, K. E. Progress in linguistics: a collection of papers. The Hague: Mouton Publishers, 1970.

CHAFFIN, R.; HERRMANN, D. J.; WINSTON, M. An empirical taxonomy of part-whole relations: effects of part-whole type on relation identification.

Language and Cognitive Processes, v. 3, n.1, p. 17-48, 1988.

CROFT, W.; CRUSE, D. A. Cognitive Linguistics. Cambridge: Cambridge University Press, 2004.

CRUSE. D. A. Lexical semantics. Cambridge: Cambridge University Press, 1986

CRUSE, D. A. On the transitivity of the part-whole relation. Journal of Linguistics, v. 15, n. 1, p. 29-38, 1979. 
FLAVELL, J. H.; MARKMAN, E. M. Cognitive development. New York: Wiley, 1983.

GERSLT, P.; PRIBBENOW, S. Midwinters, end games and body parts: a classification of part-whole relations. Int. J. Human-Computer Studies, v. 43, p. 865-889, 1995. Disponível

em:http://www.ontology.buffalo.edu/smith/courses03/tb/PribbenowMidwinters.p df. Acesso em: 12 fev. 2013.

GOTTS, N. M.; COHN, A. G. A mereological approach to representing spatial vagueness. School of Computer Studies, s.n, p. 246-255. 1995. Disponível em: http://www.qrg.northwestern.edu/papers/Files/qr-

workshops/QR95/Gotts_poster_1995_Mereological_Approach_Spatial_Vaguen ess.pdf. Acesso em: 5 abr. $201 \overline{3}$.

GUIZZARDI, G. The problem of transitivity of part-whole relations in conceptual modeling revisited. 2009. Disponível em:

http://www.inf.ufes.br/ gguizzardi/CAISE2009CR.pdf. Acesso em: 6 abr. 2013.

HJORLAND, B. Semantics and knowlegde organization. Annual Review of Information Science and Technology, v. 41, n.1, p. 367-405, 2007.

JANSEN, L. Categories: the Top-Level Ontology. In: SMITH, B.; MUNN, K. (Ed.). Applied Ontology: an introduction. Frankfurt: Ontos Verlag, 2008. p. 173- 196.

JOHANSSON, I. On the transitivity of the parthood relations, 2004. Disponivel em: http://hem.passagen.se/ijohansson/function2.PDF. Acesso em:12 maio 2018.

KEET, C. M.; ARTALE, A. Representing and reasoning over a taxonomy of part-whole relations. Applied Ontology, v. 3, n. 1, p. 91-110, 2008.

KHOO, C. S. G.; NA, J. Semantic relations in Information Science. Annual Review of Information Science and Technology, v. 40, n.1, p.157-228, 2006.

LEONARD, H. S.; GOODMAN, N. The calculus of individuals and its uses. Journal of Symbolic Logic, v.5, n. 2, p. 45-55, 1940.

LEŚNIEWSKI, S. Foundations of the general theory of Sets I. In: LEŚNIEWSKI, S., SURMA, S. J.; SRZEDNICKI, J.; BARNETT, D. I.; RICKEY, F. V. (Ed.). Collected Works. Dordrecht: Kluwer, p. 129-173, 1992.

LYONS, J. Semantics: volume 1. Cambridge: Cambridge University Press. 1977.

LYONS, J. Introduction to theoretical linguistics. Cambridge: Cambridge University Press, 1968. 
MILLER, G. A.; JOHNSON-LAIRD, P. N. Language and perception. Cambridge: Belknap Press, 1976.

MORRIS, M. An introduction to the Philosophy of Language. Cambridge: Cambridge University Press, 2007.

MURPHY, M. L. Semantic relations and the lexicon: antonymy, synonymy, and other paradigms. Cambridge: Cambridge University Press, 2003.

PRIBBENOW, S. Meronymic relationships: from classical mereology to complex part-whole relations. In: GREEN, R.; BEAN, C.A.; MYAENG, S.H. (eds.). The semantic of the relationships: an interdisciplinary perspective. Dordrecht: Kluwer Academic Publishers, 2002, p. 35-50.

RESCHER, N. Axioms for the part-whole relation. Philosophical Studies, v. 6 , p. 8-11, 1955.

SCHULZ, S.; STENZHORN, H.; BOEKER, M.; SMITH, B. Strenghts and limitations of formal ontologies in the biomedical domain. Reciis-Electronic Journal of Communication, Information and Innovation in Health, v. 3, n. 1, p. 31-45, 2009.

SIMONS, P. Parts: a study in ontology. Oxford: Oxford University Press, 1987.

SMITH, B. Ontology and Logistic analysis of reality. 1993. Disponível em: http://ontology.buffalo.edu/smith/articles/Olar.pdf. Acesso em: 3 dez. 2009.

SMITH, B.; KUNNE, W. Parts and moments: studies in logic and formal ontology. Munich: Philosophia Verlag, 2004.

SMITH, B.; MUNN, K. Applied ontology: an introduction. Frankfurt: Ontos Verlag, 2008.

SOUZA, R. R; TUDHOPE, D.; ALMEIDA, M. B. Towards a taxonomy of KOS: dimensions for classifying knowledge organization systems. Knowledge Organization, v. 39, n. 3, p. 179-200, 2012.

STOCK, W. G. Concepts and semantic relations in information science. Journal of the American Society for Information Science and Technology, v. 61, n. 10, p. 1951-1969, 2010.

TVERSKY, B. Where partonomies and taxonomies meet. In: TSOHATZIDIS (ed.). Meanings and prototypes: studies on linguistic categorization. London: Routledge, 1990. p. 334-344.

TVERSKY, B.; HEMENWAY, K. Objects, parts and categories. Journal of Experimental Psychology: General, v. 113, n. 2, p. 169-193, 1984. 
VARZI, A. C. Mereology. 2009. Disponível em: http://plato.stanford.edu/entries/mereology/. Acesso em: 20 dez. 2012.

VARZI, A. C. Parts, wholes, and part-whole relations: the prospects of mereotopology. Data and Knowledge Engineering, v. 20, n. 3, p. 259-286, 1996.

VARZI, A. C. A note on the transitivity of parthood. Applied Ontology, v.1, n. 2, p. 141-146, 2006.

WANNER, L. Lexical functions in lexicography and natural language processing. Amsterdan: John Benjamins Publishing, 1996.

WELLER, K.; STOCK, W. G. Transitive meronymy: automatic concept-based query expansion using weighted transitive part-whole relations. Information Wissenschaft \& Praxis, v. 59, n. 3, p. 165-170, 2008.

WINSTON, M. E.; CHAFFIN, R.; HERRMANN, D. A taxonomy of part-whole relations. Cognitive Science, v. 11, p. 417-444, 1987.

\title{
A THEORETICAL INVESTIGATION ABOUT PART- WHOLE SEMANTIC RELATIONS AND ITS APPLICATION IN KNOWLEDGE ORGANIZATION SYSTEMS
}

\begin{abstract}
Introduction: Semantic relationships are crucial devices for Knowledge Representation, a field of research that have received more and more attention, mainly with the advent of Semantic Web. Information Science has a long history of studying and using semantic relationships in the several contexts in which Knowledge Organization Systems (KOS) are designed and developed. However, there are a few detailed research about the semantic relationship of whole-part, also known as partitive relationship. Objective: This article aims to present a well-grounded description about the partitive relationship, from a interdisciplinary approach including its linguistic and philosophical roots. Methodology: To reach our goals, we describe and characterize two main forms through partitive relations are posed - the meronym, non-formal and linguistic in nature; the mereology, formal and logical in nature - distinguishing them by the property of transitivity. Results: From such distinction, we suggest a pragmatic criterion to decide which approach of partitive relations is suitable to build ontologies and thesauri. Conclusions: We conclude that, formal relationships are, as a matter of fact, more restrict relations, and one can even say that they are included in no-formal relationships. However, the use of formal relations in ontologies requires attention to questions sometimes neglected by information scientists.
\end{abstract}

Descriptors: Ontologies, Semantic relationships, Part-whole relationships. 


\title{
UNA INVESTIGACIÓN TEÓRICA SOBRE RELACIONES SEMÁNTICAS PARTITIVAS Y SU APLICACIÓN EN SISTEMAS DE ORGANIZACIÓN DEL CONOCIMIENTO.
}

\begin{abstract}
RESUMEN
Introducción: Las relaciones semánticas son esenciales para la Representación del Conocimiento, un campo de investigación cada vez más relevante, a partir del advenimiento de la Web Semántica. La Ciencia de la Información tiene un largo historial de estudio y uso de relaciones semánticas en los diversos contextos en que Sistemas de Organización del Conocimiento (SOCs) son diseñados y construidos. Sin embargo, son pocos los estudios detallados sobre la relación semántica parte-todo, también conocida como relación partitiva. Objetivo: el presente artículo objetiva una descripción bien fundamentada de la relación partitiva, a partir de abordaje interdisciplinario que envuelve sus orígenes lingüística y filosófica. Metodología: para alcanzar el objetivo propuesto, se describen y se caracterizan las principales formas por las cuales relaciones partitivas se manifiestan - la meronimia, no formal y lingüística; y la mereología, formal y lógica, distinguiendo por la propiedad de la transitividad. Resultados: a partir de la distinción mencionada, se sugiere criterio pragmático al decidir qué abordaje de relación partitiva es adecuada cuando se construyen ontologías y tesauros. Conclusiones: se concluye que las relaciones formales son, de hecho, más estrictas, y se puede afirmar que están incluidas en las relaciones no formales, pero que el uso de las relaciones formales en ontologías requiere cuidados muchas veces descuidados por profesional de la Ciencia de la Información.
\end{abstract}

Descriptores: Ontologías. Relaciones semánticas. Relaciones parte-todo. 